\title{
Spin-orbit coupling and chaotic rotation for eccentric coorbital bodies
}

\author{
Adrien Leleu ${ }^{1}$, Philippe Robutel ${ }^{1}$ and Alexandre C. M. Correia ${ }^{2,1}$ \\ ${ }^{1}$ IMCCE, Observatoire de Paris, CNRS, UPMC Univ. Paris 06, Univ. Lille 1, \\ 77Av.Denfert-Rochereau, 75014 Paris, France \\ email: aleleu@imcce.fr, robutel@imcce.fr \\ ${ }^{2}$ Departamento de Física, I3N, Universidade de Aveiro, Campus de Santiago, 3810-193 Aveiro, \\ Portugal \\ email: correia@ua.pt
}

\begin{abstract}
The presence of a co-orbital companion induces the splitting of the well known Keplerian spin-orbit resonances. It leads to chaotic rotation when those resonances overlap.
\end{abstract}

Keywords. celestial mechanics, coorbitals, rotation, spin-orbit resonance

\section{Introduction and Notations}

Given an asymmetric body on a circular orbit, denoting $\theta$ its rotation angle in the plane with respect to the inertial frame, the only possible spin-orbit resonance is the synchronous one $\dot{\theta}=n, n$ being the mean motion of the orbit. On an Keplerian eccentric orbit, Wisdom et al. (1984) showed that there is a whole family of spin-orbit eccentric resonances, the main ones being $\dot{\theta}=p n / 2$ where $p$ is an integer. In 2013, Correia and Robutel showed that in the circular case, the presence of a coorbital companion induced a splitting of the synchronous resonance, forming a family of co-orbital spin-orbit resonances of the form $\dot{\theta}=n \pm k \nu / 2, \nu$ being the libration frequency in the coorbital resonance. Inside this resonance, the difference of the mean anomaly of the two coorbitals, denoted by $\zeta$, librates around a value close to $\pm \pi / 3$ (around the L4 or L5 Lagrangian equilibrium - tadpole configuration), around $\pi$ (encompassing L3, L4 and L5 - horseshoe configuration) or 0 (quasi-satellite) configuration. We generalize the results of Correia and Robutel (2013) from the case of circular co-orbital orbits to eccentric ones.

\section{Rotation}

The rotation angle $\theta$ satisfies the differential equation:

$$
\ddot{\theta}+\frac{\sigma^{2}}{2}\left(\frac{a}{r}\right)^{3} \sin 2(\theta-f)=0, \text { with } \sigma=n \sqrt{\frac{3(B-A)}{C}},
$$

where $A<B<C$ are the internal momenta of the body, $(r, f)$ the polar coordinates of the center of the studied body and $a$ its instantaneous semi-major axis.

Let us consider that the orbit is quasi-periodic. As a consequence, the elliptic elements of the body can be expended in Fourier series whose frequencies are the fundamental frequencies of the planetary system. In other words the time-dependent quantity $\left(\frac{a}{r}\right)^{3} e^{i 2 f}$ that appears in equation (2.1) reads:

$$
\left(\frac{a}{r}\right)^{3} e^{i 2 f}=\sum_{j \geqslant 0} \rho_{j} e^{\left(i \eta_{j} t+\phi_{j}\right)} .
$$



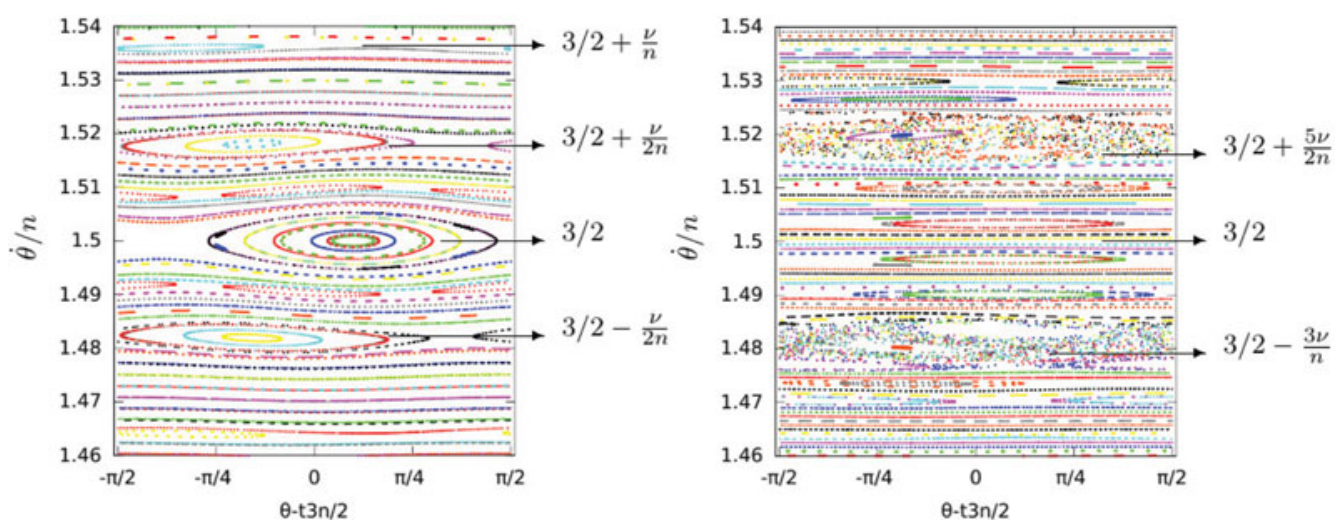

Figure 1. Poincaré surface of section in the plane $\left(\theta-t \frac{3 n}{2}, \dot{\theta} / n\right)$ near the $3 / 2$ spin-orbit eccentric resonance. (left): $\zeta_{\max }-\zeta_{\min }=35^{\circ}$ - tadpole configuration. (right): $\zeta_{\max }-\zeta_{\min }=336^{\circ}$ horseshoe configuration.

Where $\eta_{j}$ are linear combinations with integer coefficients of the fundamental frequencies of the orbital motion (here $n$ and $\nu$ ) and $\phi_{j}$ their phases. Thus (2.1) becomes:

$$
\ddot{\theta}=-\frac{\sigma^{2}}{2} \sum_{j \geqslant 0} \rho_{j} \sin \left(2 \theta+\eta_{j} t+\phi_{j}\right) .
$$

For a Keplerian circular orbit, the only spin orbit resonance possible is the synchronous one, since $\rho_{0}=1, \eta_{0}=2 n$, and $\rho_{j}=\eta_{j}=0$ for $j>0$. In the general Keplerian case we have the spin-orbit eccentric resonances, $\eta_{j}=p n$ and the $\rho_{j}$ are the Hansen coefficients $X_{p}^{-3,2}(e)$ (see Wisdom et al.). For the circular coorbital case, Correia and Robutel (2013) showed that a whole family results from the splitting of the synchronous resonance of the form $\eta_{j}=2 n \pm k \nu$. For small amplitudes of libration around L4 or L5 (tadpole), the width of the resonant island decreases as $k$ increases.

In the eccentric coorbital case, each eccentric spin-orbit resonance of the Keplerian case splits in resonant multiplets which are centred in $\dot{\theta}=p n / 2 \pm k \nu / 2$. For relatively low amplitude of libration of $\zeta$, the width of the resonant island decreases as $k$ increases, see Figure 1 (left). But for higher amplitude, especially for horseshoe orbit, the main resonant island may not be located at $k=0$. In Figure 1 (right), the main islands are located at $\dot{\theta}=3 n / 2 \pm 5 \nu / 2$ and $\dot{\theta}=3 n / 2 \pm 6 \nu / 2$. These islands overlap, giving rise to chaotic motion for the spin, while the island located at $\dot{\theta}=3 n / 2$ is much thinner.

\section{Conclusion}

The coorbital spin-orbit resonances populate the phase space between the eccentric resonances. Generalised chaotic rotation can be achieved when harmonics of co-orbital spin-orbit resonances overlap each other, which is a different mechanism than the one described by Wisdom et al. (1984), where the eccentricity harmonics overlap.

\section{References}

Correia, A. C. M. \& Robutel, P. 2013, AJ, 779, 20

Wisdom, J., Peale, S. J., \& Mignard, F. 1984, Icarus, 58, 137 